\title{
Análise dos fatores críticos e semicríticos na gestão dos resíduos de um hemocentro do Nordeste do Brasil
}

Analysis of critical and semi-critical factors in waste management in a blood center in Northeastern Brazil

Análisis de factores críticos y semicríticos en la gestión de residuos de un centro de sangre en el Noreste de Brasil

Milenna Freitas Santana

ORCID: https://orcid.org/0000-0002-7349-4107

Universidade Tiradentes, Brasil

E-mail: milennasantana@hotmail.com

Angela Maria Melo Sá Barros

ORCID: https://orcid.org/0000-0003-4087-3247

Universidade Federal do Rio de Janeiro, Brasil

E-mail: angelsamelo@hotmail.com

Carla Viviane Freitas de Jesus

ORCID: https://orcid.org/0000-0002-7775-6610 Universidade Tiradentes, Brasil

E-mail: carlavfj@gmail.com

Isabelle Borges Primo de Carvalho

ORCID: https://orcid.org/0000-0002-6789-8498 Universidade Tiradentes, Brasil

E-mail: isabelle@laboclinica.med.br

Rute Nascimento da Silva

ORCID: https://orcid.org/0000-0002-2719-1623

Universidade Tiradentes, Brasil E-mail: silva_rute@hotmail.com

Ruth Cristini Torres

ORCID: https://orcid.org/0000-0002-8664-192X Instituto de Hematologia e Hemoterapia de Sergipe, Brasil E-mail: ruthcristini@gmail.com

\begin{abstract}
Resumo
O manejo e o descarte incorreto dos Resíduos de Serviço de Saúde (RSS) podem causar enchentes, acumulação significativa de água e aparecimento de vetores transmissores de doenças. Os RSS são produzidos em hospitais, hemocentros, laboratório, consultórios, entre outros. Objetivou-se constatar os fatores críticos e semicríticos na gestão dos resíduos em um Hemocentro do Nordeste do Brasil. Trata-se de estudo qualitativo, de caráter descritivo e exploratório. Foi utilizado questionário semiestruturado direcionado para a avaliação do sistema de gestão, o manejo
\end{abstract}


dos resíduos comuns, biológicos e químicos, segregação, identificação, acondicionamento, coleta e o transporte interno, armazenamento externo, seu tratamento e disposição. O diagnóstico situacional constatou os fatores críticos e semicríticos na gestão dos resíduos no cenário do estudo. Quanto aos fatores críticos correspondentes aos aspectos ambientais, $40 \%$ atenderam as normas com restrições, sendo eles os efluentes líquidos e o sistema de gestão, enquanto $60 \%$ atenderam conforme as leis preconizadas, os quais são os resíduos comuns, químicos e biológicos. Quanto ao manejo dos resíduos tratando-se de fatores críticos, 66,6\% não atenderam as normas vigentes. Constatou-se a necessidade da implementação dos procedimentos adequados, bem como a educação permanente nos serviços de saúde, entrosando os profissionais, com o objetivo de adequar o manejo dos resíduos e dos processos, quanto à melhoria na infraestrutura fisica relacionada ao abrigo interno e externo dos RSS, visando garantia de espaço suficiente e minimização de riscos de contaminação de pessoas e do ambiente. Os dados obtidos evidenciaram resultados satisfatórios para a instituição acerca do descarte dos RSS.

Palavras-chave: Resíduos de serviço de saúde; Descarte; Hemocentro.

\begin{abstract}
The incorrect handling and disposal of Health Service Waste (HSW) can cause flooding, significant water accumulation and the appearance of disease-transmitting vectors. HSW are produced in hospitals, blood centers, laboratories, offices, among others. The objective was to verify the critical and semi-critical factors in the management of waste in a Hemocenter in the Northeast of Brazil. This is a qualitative, descriptive and exploratory study. A semi-structured questionnaire was used to assess the management system, the management of common, biological and chemical waste, segregation, identification, conditioning, collection and internal transport, external storage, its treatment and disposal. The situational diagnosis found the critical and semi-critical factors in waste management in the study scenario. As for the critical factors corresponding to the environmental aspects, $40 \%$ complied with the standards with restrictions, being the liquid effluents and the management system, while $60 \%$ complied with the recommended laws, which are the common, chemical and biological residues. As for the management of waste in the case of critical factors, $66.6 \%$ did not meet the current standards. There was a need for the implementation of appropriate procedures, as well as permanent education in health services, involving professionals, with the aim of adapting the management of waste and processes, regarding the improvement in physical infrastructure related to internal and external shelter of HSW, aiming at guaranteeing sufficient space and minimizing risks of contamination of people and the environment. The data obtained showed satisfactory results for the institution regarding the disposal of HSW.
\end{abstract}

Keywords: Health service waste; Disposal; Blood center.

\title{
Resumen
}

El manejo y disposición incorrecta de los Residuos del Servicio de Salud (RSS) puede provocar inundaciones, acumulación significativa de agua y la aparición de vectores transmisores de enfermedades. Los RSS se producen en hospitales, centros de sangre, laboratorios, oficinas, entre otros. El objetivo fue verificar los factores críticos y semicríticos en el manejo de residuos en un Hemocentro en el Nordeste de Brasil. Se trata de un estudio cualitativo, descriptivo y exploratorio. Se utilizó un cuestionario semiestructurado para evaluar el sistema de gestión, la gestión de residuos comunes, biológicos y químicos, segregación, identificación, acondicionamiento, recogida y transporte interno, almacenamiento externo, su tratamiento y disposición. El diagnóstico situacional encontró los factores críticos y semicríticos en la gestión de residuos en el escenario de estudio. En cuanto a los factores críticos correspondientes a los aspectos ambientales, el $40 \%$ cumplió con las normas con restricciones, siendo los efluentes líquidos y el sistema de gestión, mientras que el $60 \%$ cumplió con las leyes recomendadas, que son los residuos comunes, químicos y biológicos. En cuanto al manejo de residuos en el caso de factores críticos, el 66,6\% no cumplió con los estándares vigentes. Se requería la implementación de procedimientos adecuados, así como la educación permanente en los servicios de salud, involucrando a los profesionales, con el objetivo de adecuar la gestión de residuos y procesos, en cuanto a la mejora de la infraestructura física relacionada con el albergue interno y externo. De RSS , con el objetivo de garantizar un espacio suficiente y minimizar los riesgos de contaminación de las personas y el medio ambiente. Los datos obtenidos arrojaron resultados satisfactorios para la institución en cuanto a la disposición de RSS.

Palabras clave: Residuos de servicios de salud; Disposición; Centro de sangre.

\section{Introdução}

Atualmente, nos deparamos com a falta de conscientização humana, onde somos vistos como a "sociedade do lixo", por causar altos níveis de desperdícios e pelo grau de evolução tecnológica e industrial, o que resulta um enorme problema ambiental (Castro, Guimarães, Lima, Lopes \& Chaves, 2014). Com o crescimento rápido da população e das cidades vários problemas foram criados para o meio ambiente e devido a suas consequências foram necessárias medidas de ações de políticas públicas para minimizar a destruição do meio ambiente e dos lixos dos centros urbanos. Entretanto, com a produção de resíduos domésticos, sólidos, hospitalares, laboratoriais, industriais, pelas atividades dos seres humanos necessitam de um destino preciso 
afim de que não venha trazer transtornos para o ecossistema global como um todo e destruição da biodiversidade em todas as suas formas (Lovelock, 2006).

Diversos fatores contribuem para o crescimento da geração de Resíduos de Serviço de Saúde (RSS), tais como: materiais descartáveis, atenção básica médica, o crescimento da população da terceira idade que precisa de mais atendimento no serviço de saúde assim como as instituições de saúde existentes entre ambulatórios, hospitais, programa de suade familiar, pronto socorro (Schneider, 2004). Neste cenário, é importante destacar que o gerenciamento adequado dos RSS contribui para a segurança ocupacional dos profissionais que os manipulam, desde a segregação até o destino final, assim como para a saúde pública e a preservação do meio ambiente (Pereira \& Kozusny-Andreani, 2020).

Aconteceu no ano de 1993, no território brasileiro quando a Associação Brasileira de Normas Técnicas (ABNT) começou a publicar normas e legislação direcionadas para os resíduos de saúde e continuam sendo trabalhadas até hoje com o intuito de melhorar cada vez mais a gestão dos resíduos como às NBR 12.235 (ABNT, 1992), NBR 12.810 (ABNT, 1993), NBR 13853 (ABNT, 1997), NBR 7.500 (ABNT, 2000a), NBR 9.191 (ABNT, 2000b), NBR 14.652 (ABNT, 2001a), NBR 14.725 (ABNT, 2001b), NBR 10.004 (ABNT, 2004), NBR 15.849 (ABNT, 2010; Godecke, Naime \& Figueiredo, 2012).

Com base na Resolução da Diretoria Colegiada, da Agência Nacional de Vigilância Sanitária no 33 (Brasil, 2005) e nº 306 (Brasil, 2004) e a norma do Conselho Nacional do Meio Ambiente (CONAMA) Resolução nº 358 (Brasil, 2005), que procedimentos operacionais relacionados ao gerenciamento de RSS tem sido de forma gradativa implantada e implementada, no entanto os profissionais de saúde tem tido dificuldades na elaboração documento do Programa de Gerenciamento de Resíduos de Serviços de Saúde (PGRSS) (ABELPR, 2018). Faz necessário o treinamento com profissionais da área de saúde, para que sejam capacitados a concluir o serviço prestados.

O Ministério da Saúde (Brasil, 2018) através da Agencia Nacional de Vigilância Sanitária (ANVISA), afirma que os procedimentos operacionais realizados pelos Estabelecimentos Assistenciais de Saúde (EAS) envolve resíduos infectantes prejudiciais à saúde da natureza assim como a Saúde humana, tais como: sangue, hemocomponentes, perfuro cortantes, radioativos, químicos, tecidos, animais contaminados, entre outros.

De acordo com a RDC ANVISA n 222 (Brasil, 2018) e Resolução CONAMA nº 358 (Brasil, 2005), os RSS são classificados em cinco grupos: A, B, C, D e E. Grupo A (resíduos biológicos), com presença de material biológico que, em função de suas características, pode oferecer algum tipo de risco de contaminação; Grupo B (resíduos químicos) que, em sua composição, contém substâncias químicas que podem representar risco ao meio ambiente e à saúde pública, por suas características de inflamabilidade, corrosividade, toxicidade e reatividade; Grupo C (rejeitos radioativos), resíduos contendo radionuclídeos em concentrações superiores às estabelecidas pela Comissão Nacional de Energia Nuclear (CNEN), sem a possibilidade de reaproveitamento; Grupo D (resíduos comuns), que não apresentam nenhuma das características dos grupos citados anteriormente; e Grupo E (perfuro cortantes), abrangendo todos os materiais perfurantes, cortantes e escarificantes (Figura 1).

Os RSS são identificados nos recipientes onde os resíduos serão dispostos. Esta prática consiste no conjunto de medidas que permite o reconhecimento dos resíduos contido nos sacos e recipientes, fornecendo informações ao correto manejo dos RSS, conforme demonstrado na (Figura 1). 
Figura 1. Símbolos de identificação dos grupos de resíduos de serviço de saúde

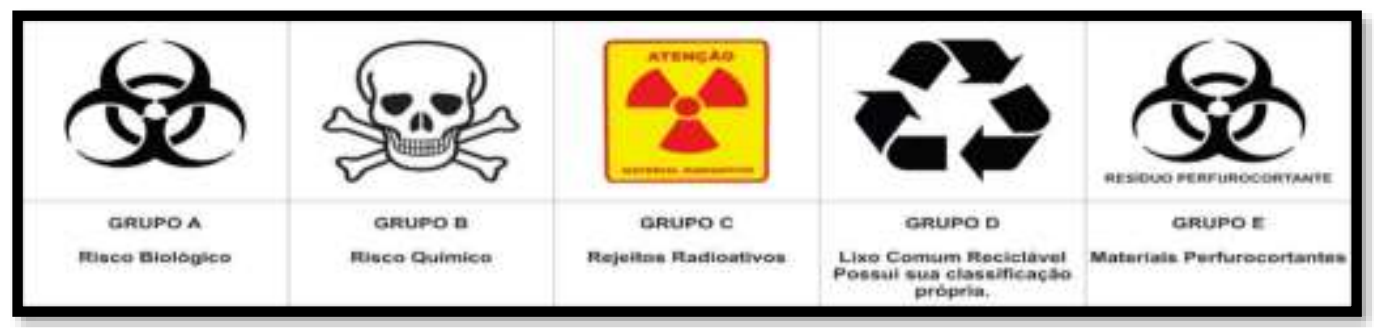

Fonte: Grupo de Pesquisa e Extensão em Segurança e Sustentabilidade em Saúde. Escola de Enfermagem Anna Nery/UFRJ. https://gpeseg.blogspot.com/2017/08/gpeseg-explica-segregacao-e-descarte-de.html

Os EAS são responsáveis por manter de maneira conforme toda a produção de RSS por eles produzidos, atendendo as normas e legislações vigentes, desde a sua geração até a sua destinação final, afim de prevenir a saúde da população e do meio ambiente (Brasil, 2018).

É inquestionável a necessidade de implantar PGSS nos diversos estabelecimentos de saúde, não apenas investindo na organização e estimatização dessas fontes geradoras, mas, fundamentalmente, mediante o despertar de uma consciência humana e coletiva quanto à responsabilidade com a própria vida humana e com o ambiente. Os resíduos de serviços de saúde são produzidos em hospitais, hemocentros, laboratório, consultórios, clínica veterinária, entre outros (Nascimento, 2001). Gonçalves et al. (2020), reforçam a importância da capacitação de todos os envolvidos no âmbito dos RSS, visando a gestão correta dos resíduos citados, respeitando as normas de biossegurança.

O PGSS deve contemplar o planejamento, implantação e monitoramento das atividades que possam causar exposição ao risco ocupacional dos profissionais, proporcionar a segurança dos mesmos e dos usuários dos serviços de saúde, como também apresentar medidas para que não ocorram impactos ambientais provenientes do descarte dos RSS, abrangendo inclusive a normas biossegurança (Uehara, Veiga \& Takayanagui, 2019). Assim, o objetivo deste estudo foi de constatar os fatores críticos e semicríticos na gestão dos resíduos de um Hemocentro da região Nordeste do Brasil.

\section{Metodologia}

A pesquisa foi realizada nos setores de Hemocentro do Nordeste do Brasil. Trata-se de estudo qualitativo, de caráter descritivo e exploratório.

Foi utilizado questionário quantitativo e qualitativo com perguntas fechadas, abertas e semiabertas direcionadas ao gerente dos RSS do hemocentro coordenador (HC), avaliando o sistema de gestão, o manejo dos resíduos comuns, biológicos e químicos, segregação, identificação, acondicionamento, coleta e o transporte interno, armazenamento externo, seu tratamento e disposição.

Com as informações foi gerado um banco de dados na planilha EXCEL 2017 e os resultados foram apresentados em tabelas e figuras. Participaram dessa pesquisa os profissionais que trabalham com os RSS neste hemocentro, sendo eles um técnico contábil, uma farmacêutica, uma analista de patologia clínica, uma assessora técnica, dois enfermeiros, um técnico em laboratório, dois técnicos em enfermagem.

O Hemocentro tem por finalidade prestar serviços relativos à coleta, processamento, estocagem, distribuição e aplicação do sangue, seus componentes e hemoderivados. Os serviços de saúde prestados pela Fundação são organizados em conformidade com as diretrizes e normas do Sistema Único de Saúde - SUS, devendo servir de campo de prática para ensino e pesquisa na área da saúde. Conforme as atividades executadas no Hemocentro, os RSS são classificados de acordo com a Tabela 1. 
Tabela 1. Classificação dos Resíduos de Serviços de Saúde gerados no Hemocentro Coordenador de Sergipe.

\begin{tabular}{|c|r|}
\hline CLASSIFICAÇ̃̃O & DESCRIÇÂO \\
\hline Grupo A & $\begin{array}{r}\text { Bolsas de sangue para serem transfundidas, ou com sorologia reagente, tubos de } \\
\text { ensaio contendo restos de amostras de sangue, luvas de procedimentos, algodão. }\end{array}$ \\
\hline Grupo B & Frascos de desinfetantes e saneantes, frascos de reagentes, medicamentos. \\
\hline Grupo C & Não é produzido. \\
\hline Grupo D & $\begin{array}{r}\text { Papel, restos de alimentos, copos descartáveis, resíduos de varrição, podas, } \\
\text { flores e jardins, resíduos provenientes de áreas administrativas. }\end{array}$ \\
\hline Grupo E & Agulhas, seringas, escalpes, lancetas, microcuvetas. \\
\hline
\end{tabular}

Fonte: Autores.

O cenário do estudo possui área total do terreno: em torno de 13.410,13 $\mathrm{m}^{2}$ Quantidade de prédios: 05 numa área total construída: aproximadamente 4.155,00 m², caracterizado pelos setores recepção, coleta, transfusão, ambulatório técnico, laboratórios, área administrativa e Unidade de Tratamento de Resíduos.

\section{Resultados e Discussão}

O diagnóstico situacional constatou os fatores críticos e semicríticos na gestão dos resíduos do Hemocentro em questão. Quanto aos fatores críticos correspondentes aos aspectos ambientais, $40 \%$ (2) atenderam as normas com restrições, sendo eles os efluentes líquidos e o sistema de gestão, enquanto $60 \%$ (3) atenderam conforme as leis preconizadas, os quais são os resíduos comuns, químicos e biológicos (Figura 2).

Figura 2. Aspectos críticos ambientais no PGRSS do Hemocentro no período de fevereiro a abril de 2018.

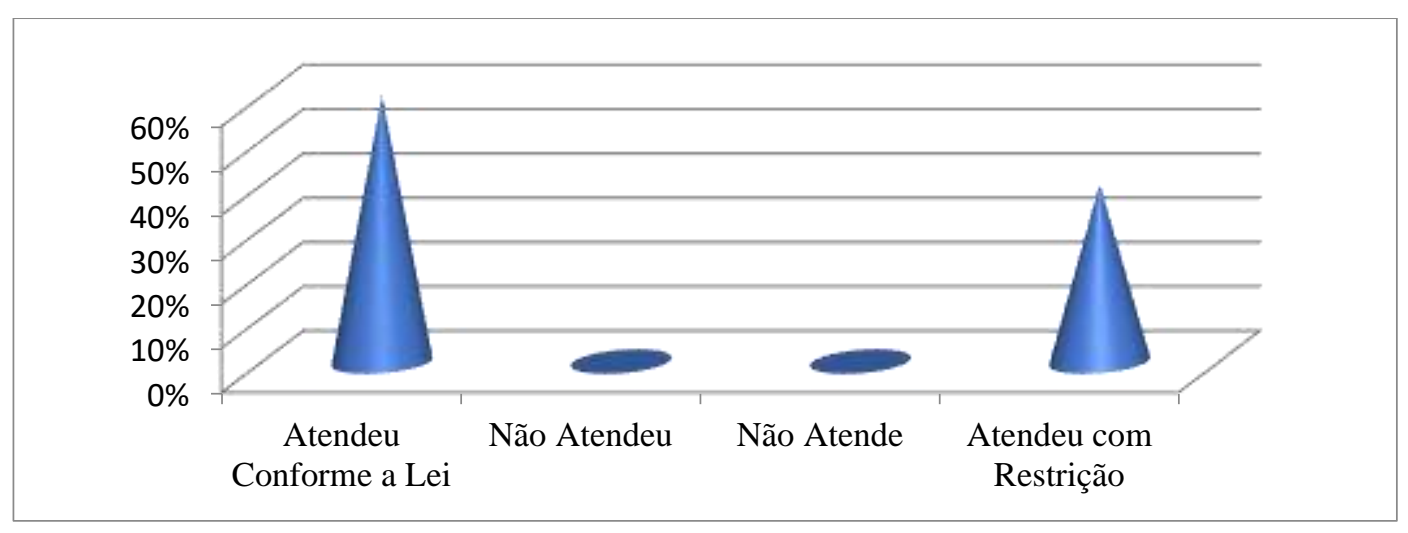

Fonte: Autores.

Efluentes de estabelecimentos assistenciais de saúde são caracterizados como possíveis veículos de disseminação de inúmeros microrganismos patogênicos, entretanto quando não passam pelo tratamento antes de serem descartados, contaminam mananciais de água potável, tanto superficial quanto subterrânea, gerando riscos à saúde pública se atingirem o sistema de abastecimento (Vecchia, Thewes, Harb Naime \& Spilki, 2009).

Quanto ao manejo dos resíduos tratando-se de fatores críticos, 66,6\% (4) não atenderam as normas vigentes, sendo os itens da identificação, armazenamento temporário, armazenamento externo e tratamento do resíduo, enquanto 33,3\% (2) atenderam as normas com restrições, sendo estas presentes os itens da segregação, coleta e transporte interno. Os Resíduos do grupo B (Químico) têm dificuldades para o tratamento final por não haver empresas terceirizadas no Estado que faça o tratamento e destinação final (Figura 3). 
Figura 3. Manejo dos resíduos no PGRSS do Hemocentro no período de fevereiro a abril de 2018.

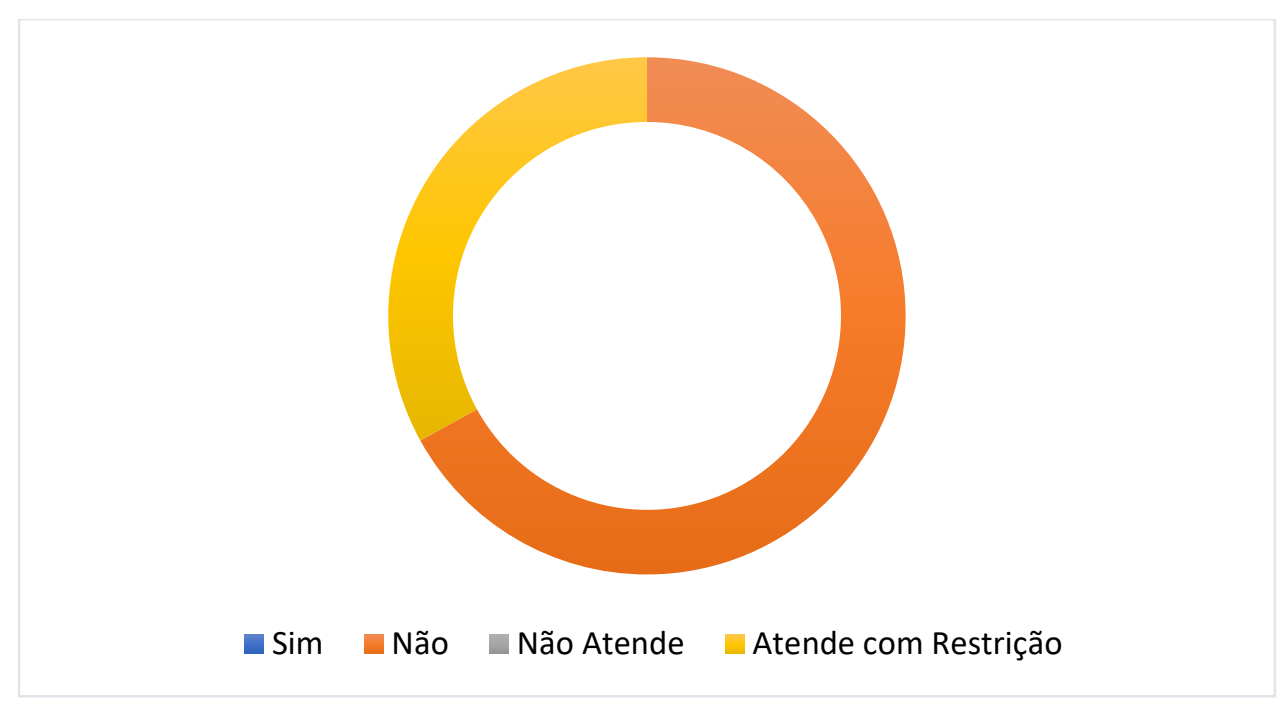

Fonte: Autores.

O manejo dos resíduos deve seguir um fluxograma de acordo com suas características, para que sejam evitados acidentes no trabalho, principalmente na fase das coletas dos RSS se a segregação for incorreta (Oliveira et al., 2018). Atualmente o manejo precário dos RSS é um problema de saúde pública, o que acarreta problemas diversos tanto para o meio ambiente como para a saúde dos indivíduos, no entanto pesquisa realizada por Gessner, Piosiadlo, Fonseca \& Larocca (2013) em uma Unidade de Saúde, demonstrou que o manejo correto na etapa da segregação e do acondicionamento faz parte dos procedimentos diários dos profissionais, no entanto estes não reconhecem o processo como um todo, sobretudo, das etapas que não são executadas em seus locais de trabalho.

O abrigo externo para RSS deve ser um local de uso exclusivo dos serviços, e dispor de área separada para os resíduos dos Grupos A, B, D e E. Sua localização deve estar de fácil acesso para o transporte externo dos resíduos oriundos da unidade geradora e também facilitar o acesso dos veículos coletores dos resíduos. Como também em área com condições seguras, evitando o acesso de pessoas e animais, de boa iluminação, ventilação e revestimentos de pisos para facilitar o processo de limpeza (Pozzetti \& Monteverde, 2017).

Quando avaliados os fatores semicríticos dos aspectos ambientais, 75\% (3) atenderam as normas com restrições, sendo o abastecimento de água, resíduos comuns recicláveis e resíduos químicos, enquanto $25 \%$ (1) não realizou a análise da qualidade, tratando-se do efluente líquido, principalmente dos laboratórios que lançam produtos químicos e biológicos na pia (Figura 4). 
Figura 4. Avaliação dos itens acerca dos aspectos ambientais dos fatores semicríticos no Hemocentro.

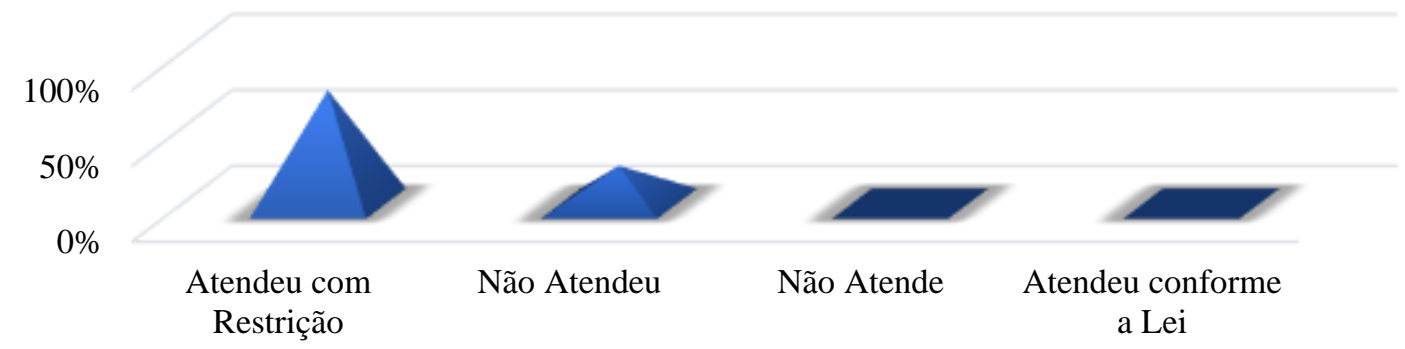

Fonte: Autores.

Outro aspecto avaliado foi o manejo de resíduos de fatores semicríticos, o qual apresentou 100\% (2) dos itens atendentes segundo as normas apresentando restrição, sendo eles a coleta e transporte interno e a disposição final dos resíduos.

Quando avaliado sobre a saúde e segurança do trabalhador no item biossegurança, foi observado que houve o atendimento com certas restrições no plano de emergência, contingência e o mapa de risco encontra-se mal elaborado (Figura $5)$.

Figura 5. Avaliação do item de saúde e segurança do trabalhador no Hemocentro.

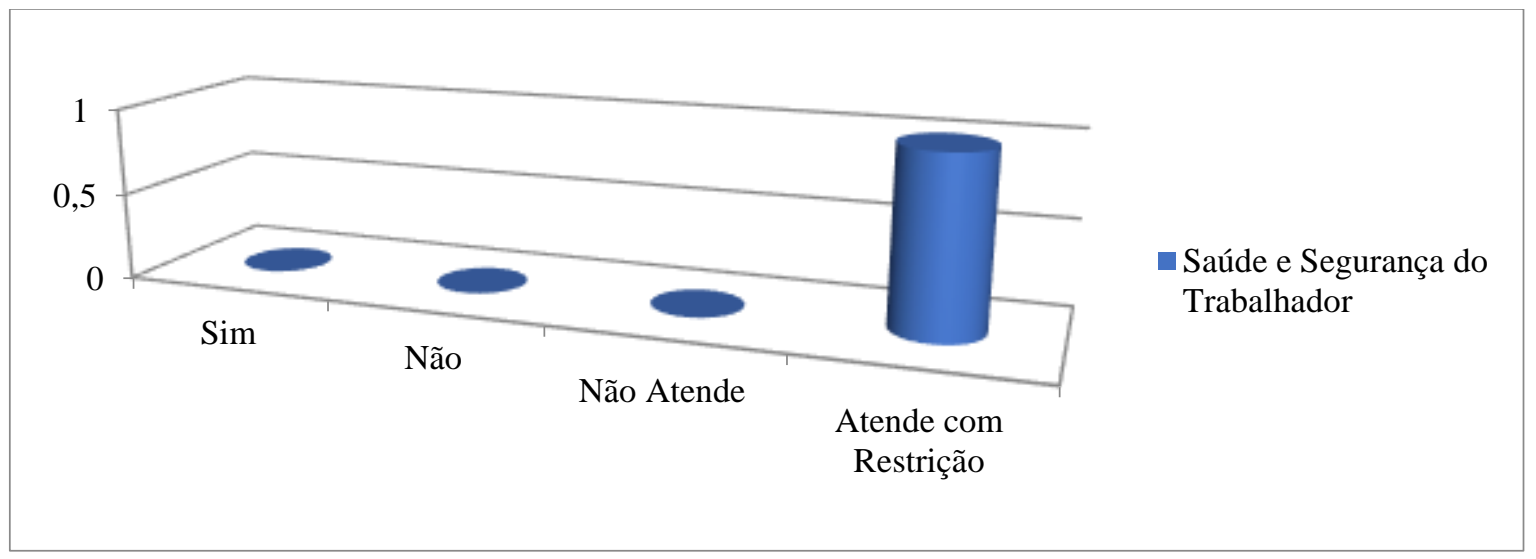

Fonte: Autores.

Os resíduos químicos de laboratório se enquadram em dois tipos básicos: o estabelecimento de rotas de gestão, focando o tratamento de correntes residuais para um descarte final adequado e/ou a recuperação de elementos de elevado valor agregado (Alberguini, Silva \& Rezende, 2003), bem como na avaliação do impacto desses programas de gestão sobre as pessoas envolvidas nos mesmos, com ênfase na percepção e assimilação de uma nova cultura comprometida com o meio ambiente e a gestão dos resíduos gerados por eles mesmos (Felisberto et al., 2008).

Baseado nas não conformidades supracitadas foi construído um plano de ação baseado nas não conformidades tendo como meta de melhoria: Adequação das instalações hidráulicas, elétricas e prediais; conhecimento e descarte correto dos efluentes líquidos; adequação dos abrigos de resíduos; sanar as não conformidades na segregação, disposição de sacos de lixo no chão, coleta interna dos resíduos comum, riscos de acidentes e quantificação de resíduo na unidade avaliada; Organizar, parcialmente, o Abrigo de Resíduo Químico (abrigo temporário); Transportar, tratar e realizar descarte final de resíduos. 
Quanto ao manejo dos resíduos de serviços de saúde, verificou-se que o Hemocentro possui um gerente responsável pelo processo do PGRSS e um técnico que trabalha com ações relacionadas a educação aos RSS. Tal fato é reforçado pelos achados de Coswosk et al. (2018), que ressaltaram a necessidade da educação continuada dos profissionais da saúde para a manutenção da qualidade do serviço, atualização do conhecimento científico, bem como para um ambiente de trabalho mais seguro.

\section{Considerações Finais}

Com base nos resultados observados, faz-se necessário a promoção e a implementação dos procedimentos adequados, vislumbrando a educação permanente nestes serviços, a partir de treinamento por toda a equipe de saúde e limpeza, entrosando os profissionais, com o objetivo de adequar o manejo dos resíduos e dos processos, quanto à melhoria na infraestrutura fisica relacionada ao abrigo interno e externo dos RSS, visando garantia de espaço suficiente e minimização de riscos de contaminação de pessoas e do ambiente.

Embora a instituição possua um PGRSS sempre é necessário dispensar maior atenção para o atendimento das etapas de manejo previstas na $\operatorname{RDC}^{\circ}{ }^{\circ} 22$, de 28 de março de 2018, bem como alternar formas de prevenção e tratamento, estipulando melhores resultados na disposição final do Programa, de acordo com as exigências técnicas e legais dos bancos de sangue e das normas legais nacionais.

Os dados obtidos pela presente pesquisa, visando análises experimentais, evidenciou resultados satisfatórios para a instituição. Faz necessária novas pesquisas sobre critérios da utilização de autoclave, a fim de reduzir ainda mais os riscos de contaminação, com o tratamento de bolsas de sangue contaminadas ou outros materiais de risco biológico, principalmente em processos manuais, tangendo às questões físicas e operacionais internas e a forma de monitoramento.

\section{Referências}

Alberguini, L. B. A., Silva, L. C., \& Rezende, M. O. O. (2003). Tratamento de resíduos químicos: guia prático para a solução dos resíduos químicos. Quim. Nova. RIMA.

Associação Brasileira de Empresas de Limpeza Pública e Resíduos - ABELPR (2018). Panorama dos resíduos sólidos no Brasil 2017. http://abrelpe.org.br/pdfs/panorama/panorama_abrelpe_2017.pdf

Associação Brasileira de Normas Técnicas - ABNT. NBR 10.004 (2004). Resíduos sólidos - Classificação.

Associação Brasileira de Normas Técnicas - ABNT. NBR 12.235 (1992). Armazenamento de resíduos sólidos perigosos.

Associação Brasileira de Normas Técnicas - ABNT. NBR 12.810 (1993). Coleta de resíduos de serviços de saúde.

Associação Brasileira de Normas Técnicas - ABNT. NBR 13.853 (1997). Coletores para resíduos de serviços de saúde perfurantes ou cortantes: requisitos e métodos de ensaio.

Associação Brasileira de Normas Técnicas - ABNT. NBR 14.652 (2001a). Coletor-transportador rodoviário de resíduos de serviços de saúde - Requisitos de construção e inspeção.

Associação Brasileira de Normas Técnicas - ABNT. NBR 14.725 (2001b). Ficha de informações de segurança de produtos químicos. Rio de Janeiro.

Associação Brasileira de Normas Técnicas - ABNT. NBR 15.849 (2010). Resíduos sólidos urbanos - Aterros sanitários de pequeno porte - Diretrizes para localização, projeto, implantação, operação e encerramento.

Associação Brasileira de Normas Técnicas - ABNT. NBR 7.500 (2000a). Símbolos de Riscos.

Associação Brasileira de Normas Técnicas - ABNT. NBR 9.191 (2000b). Classificação e Sacos Plásticos para Coleta de Lixo.

Brasil (2018). Resolução RDC $\mathrm{n}^{\circ}$ 222. Brasília-DF, Brasil: Ministério da Saúde. http://portal.anvisa.gov.br/documents/10181/3 427425/RDC_222_2018_.pdf/c5d3081d-b331-4626-8448-c9aa426ec410.

Brasil. Ministério da Saúde (2004). Resolução da Diretoria Colegiada n ${ }^{\circ}$ 306, de 7 de dezembro de 2004. Dispõe sobre o Regulamento Técnico para o gerenciamento de resíduos de serviços de saúde.

Brasil. Ministério da Saúde (2005). Resolução da Diretoria Colegiada n ${ }^{\circ}$ 33, de 25 de fevereiro de 2005. Dispõe sobre o Regulamento Técnico para o gerenciamento de resíduos de serviços de saúde. 
Research, Society and Development, v. 10, n. 5, e43410515082, 2021

(CC BY 4.0) | ISSN 2525-3409 | DOI: http://dx.doi.org/10.33448/rsd-v10i5.15082

Brasil. Ministério do Meio Ambiente (2005). Conselho Nacional do Meio Ambiente Resolução no 358 , de 29 de abril de 2005. Dispõe sobre o tratamento e a disposição final dos resíduos dos serviços de saúde e dá outras providências.

Castro, R. R., Guimarães, O. S., Lima, V. M. L., Lopes, C. D. F., \& Chaves, E. M. (2014). Gerenciamento dos resíduos de serviços de saúde em um hospital de pequeno porte. Rev Rene, 15(5): 860-8.

Coswosk, E. D., Santa Rosa, C. G., Caldeira, A. B., Silva, N. C. R., \& Rocha, J. M. (2018). Educação continuada para o profissional de saúde no gerenciamento de resíduos de Saúde. Rev. bras. anal. Clin., 50(3):288-96.

Felisberto, R., Vieira, L. O., Couto, A., Schuh, R., Albino, C. T., Libardi, D. B., \& Cunha, A. C. B. (2008). De resíduo a insumo: a construção do caminho para uma química mais limpa através de um projeto de ensino. Quím. Nova, 31(1): 174-177.

Gessner, R., Piosiadlo, L. C. M., Fonseca, R. M. G. S., \& Larocca, L. M. (2013). O Manejo dos Resíduos dos Serviços de Saúde: um problema a ser enfrentado. Cogitare Enfermagem, 18(1): 117-23.

Godecke, M. V., Naime, R. H., \& Figueiredo, J. A. S. (2012). O consumismo e a geração de resíduos sólidos urbanos no Brasil. Reget, 8(8): 1700-12.

Gonçalves, C. S., Nazari, M. T., Silva, P. L. C., Peruchin, B., Corrêa, E. K., \& Corrêa, L. B. Biossegurança em hospital veterinário: os resíduos de serviços de saúde sob a perspectiva dos geradores. Research, Society and Development, 9, (3): 1-19.

Grupo de Pesquisa e Extensão em Segurança e Sustentabilidade em Saúde (2017). Segregação e descarte de RSS. https://gpeseg.blogspot.com.br/2017/08/gpeseg-explica-segregacao-e-descarte-de.html.

Lovelock, J. E. (2006). A vingança de gaia. Intrínseca.

Nascimento, M. C. B. (2001). Seleção de sítios visando à implantação de aterros sanitários com base em critérios geológicos, geomorfológicos e hidrológicos. Dissertação de mestrado, Instituto de Geociências, Universidade de São Paulo.

Oliveira, L., et al. (2018). Fatores associados ao manejo adequado de resíduos de serviços de saúde entre profissionais de enfermagem. Revista Baiana de Enfermagem, 32; 1-11.

Pereira, R. A., \& Kozusny-Andreani, D. I. (2020). Gestão dos resíduos sólidos nas unidades básicas de saúde no Município de Guaraí, Estado do Tocantins, Brasil. Research, Society and Development, 9(9): 1-14.

Pozzetti, V. C., \& Monteverde, J. F. S. (2017). Gerenciamento ambiental e descarte do lixo hospitalar. Revista Veredas do Direito, 14(28): 195-220.

Schneider, V. E. (2004). Manual de Gerenciamento de Resíduos Sólidos de Saúde. Caxias de Sul (RS), Editoria da Universidad de Caxias do Sul, (2a ed.).

Uehara, S., Veiga, T., \& Takayanagui, A. (2019). Gerenciamento de resíduos de serviços de saúde em hospitais de Ribeirão Preto(SP), Brasil. Engenharia Sanitária e Ambiental, 24(1), 121-130.

Vecchia, A. D., Thewes, M. R., Harb Naime, R., \& Spilki, F. R. (2009). Diagnóstico sobre a situação do tratamento do esgoto hospitalar no Brasil. Rev Saúde Ambiente, 10: 65-9. 